\title{
Aggressive Angiomyxoma of the Perineum: A Rare Case Entity and Literature Review
}

Sumit Pandita ${ }^{1} \quad$ Irfan Bashir ${ }^{1} \quad$ Manish Sharma ${ }^{1} \quad$ Swarnita Sahu ${ }^{1}$

\begin{abstract}
Address for correspondence Sumit Pandita, DNB, Department of Radiation Oncology, Batra Hospital and Medical Research Centre, Tuglukabad, New Delhi 110062, India (e-mail: sumitv.2010@rediffmail.com).
\end{abstract}
Abstract
Keywords
- aggressive angiomyxoma
- radiotherapy
- hormonal therapy

Angiomyxomas are soft tissue tumors that are exceedingly rare with very few cases described in literature. They are classified into a less aggressive superficial variant and a deeper aggressive variant commonly called aggressive angiomyxomas (AA). The cross-sectional imaging, particularly magnetic resonance imaging (MRI), plays an essential role in the diagnosis and management of AA. We report a rare case of Aggressive angiomyxoma in a 28-year-old female.

\section{Introduction}

Aggressive angiomyxoma (AA) is a very rare soft tissue mesenchymal tumor, typically found in the perineum of females in reproductive age group. ${ }^{1}$ Commonly, the tumor simulates as a Bartholin's cyst, abscess or hernia because most patients complain of pain in the perineum, labia, or pelvis. ${ }^{2}$ It grows slowly as a painless solid mass with unclear borders and the AA often infiltrates the deep tissues. ${ }^{3}$ Even if the tumor infiltrates the bladder, rectum, retroperitoneum, pelvic bone, or levator ani muscle, the patients are often asymptomatic., ${ }^{4,5}$ Surgical excision is the treatment of choice for AA; however, there is a high rate of recurrence. ${ }^{6}$ The local recurrence rate is 25 to $47 \%$ after surgical excision. ${ }^{7}$ Complete surgical excision of these tumors is often difficult because of their resemblance to normal tissue. ${ }^{8}$ Although angiomyxoma belongs to a category of neoplasms considered to be radioresistant, ${ }^{9}$ adjuvant radiotherapy (RT) has been reported to be effective in preventing recurrences. ${ }^{10}$

\section{Case Report}

A 28-year-old healthy woman presented with a 4-year history of a painless swelling on the right side of labia majora. The swelling was gradually increasing in size. There was no history of discharge, difficulty in defecation or urination. On physical examination, the patient was not pale or jaundiced, and the examination of head, neck, chest, and abdomen was unremarkable. Local examination of the perineal region revealed large soft cystic swelling which was nontender on palpation. Contrast-enhanced computed tomography (CECT) scan of the pelvic region revealed a well-defined long segment heterogeneous density lesion measuring $11 \mathrm{~cm} \times 5 \mathrm{~cm}$ with central cystic areas extending from right pararectal space up to labia majora. Lesion was closely abutting the vagina with ill-defined intervening fat planes. No definitive communication with gut loop was seen. The case was discussed in the tumor board of our hospital and the patient underwent wide-local excision of the perineal mass. The gross histopathological examination of the surgical specimen showed a externally bosselated gray-brown mass measuring $12 \mathrm{~cm} \times 9 \mathrm{~cm} \times 3 \mathrm{~cm}$. Cut-section of the specimen revealed gray-white soft tissue with mucoid areas. Microscopic examination showed features of AA entrapping the adjacent skeletal muscle fibers. The deep resection margin was involved. Sections from the skin flap were also involved by tumor. The routine hematoxylin and eosin-stained sections examined from the tumor showed low to moderate cellularity with small, stellate, and spindle cells embedded in loosely collagenous myxedematous matrix containing scattered vessels of varying calibre. The lesion was seen infiltrating between skeletal muscle bundles. No mitosis was seen ( - Fig. 1A). On immunohistochemistry, tumor cells were strongly posi-

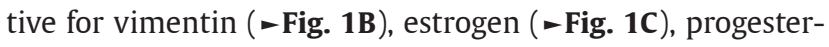
one ( - Fig. 1D), desmin ( - Fig. 1E), and negative for CD 34 ( - Fig. 1F), smooth muscle antigen (SMA), and S-100.

In view of positive margin status, patient was treated with adjuvant RT to the postoperative bed to a dose of $50 \mathrm{~Gy}$

\section{received}

September 20, 2019

accepted

October 15, 2019
DOI https://doi.org/

$10.1055 / \mathrm{s}-0039-3400711$

ISSN 2454-6798.
C2019 Spring Hope Cancer Foundation \& Young Oncologist Group of Asia
License terms

(우 (1) $\ominus \circledast$ 

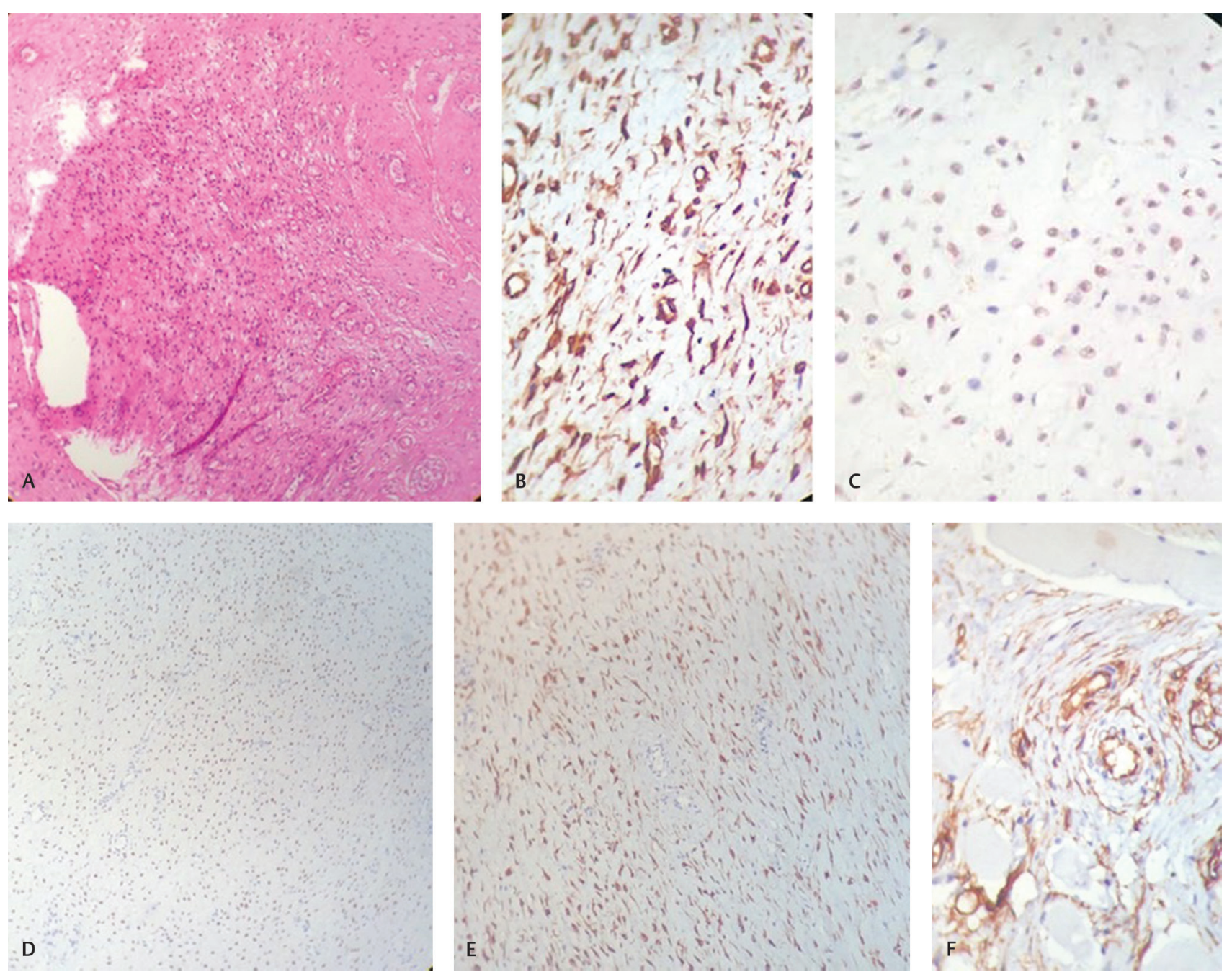

Fig. 1 (A) Section showing low-to-moderate cellularity with small, stellate, and spindled cells embedded in loosely collagenous myxedematous matrix containing scattered vessels of varying calibre. No mitosis seen (H\&E, $\times 40)$. (B) Immunohistochemical analysis showed that tumor cells are positive for vimentin. (C) Estrogen receptor immunostatin is positive in tumor cells. (D) Progesterone receptor (PR) immunostatin is positive in tumor cells. (E) Desmin immunostatin is positive in tumor cells. (F) Tumor cells are negative for CD-34 marker.

in 25 fractions, 2 Gy per fraction, one fraction per day over a period of 5 weeks. A volumetric modulated arc therapy (VMAT) plan was generated to optimize dose distribution delivering maximum dose to the target volume and doses to the organs at risk were within acceptable limits ( - Fig. 2). Treatment was delivered under daily image guidance.

During treatment, the patient experienced grade- 1 dermatitis of the right intragluteal fold which was managed symptomatically. After completion of RT, patient was started on adjuvant tamoxifen $20 \mathrm{mg}$ tablet daily. At 3-month follow-up, the skin reactions were healed and CECT pelvis showed postradiotherapy changes with no evidence of any recurrence.

\section{Discussion}

Although AA is a rare entity, it is important to be aware of its clinical features. The initial presentation is often a painless, asymptomatic perineal, or vulval nodule. Overlying skin may appear normal, and clinicians may mistake AA for a benign entity. The mass may be discovered upon routine gynecologic examination or by the patient herself. Because of the rarity of the AA, preoperative diagnosis is often difficult and a lot of cases are diagnosed on histological findings after the surgical excision. ${ }^{11,12}$ Macroscopically the surface of tumor is smooth, partially or completely encapsulated, lobular, with borders that are not well circumscribed. ${ }^{11}$ Histologically the tumor demonstrates stellate-to-spindle shaped cells distributed sparsely throughout the myxoid stroma and aggregated around blood vessels. Mitotic figures are infrequent and not atypical, and there is no necrosis within the tumor. ${ }^{13,14}$ Immunohistochemistry commonly is positive for CD 34, SMA, desmin, vimentin, estrogen, and progesterone receptors and is typically negative for S- $100 .{ }^{14,15}$ For timely diagnosis, it is important to have a high index of suspicion for AA. Misdiagnosis rates have been reported as high as $82 \%$. Fourteen radiological imaging is critical in assessing extent and surgical approach and in surveillance for recurrences. Multiple imaging modalities have been used to evaluate AA preoperatively including ultrasonography (USG), CT scan (as in the 


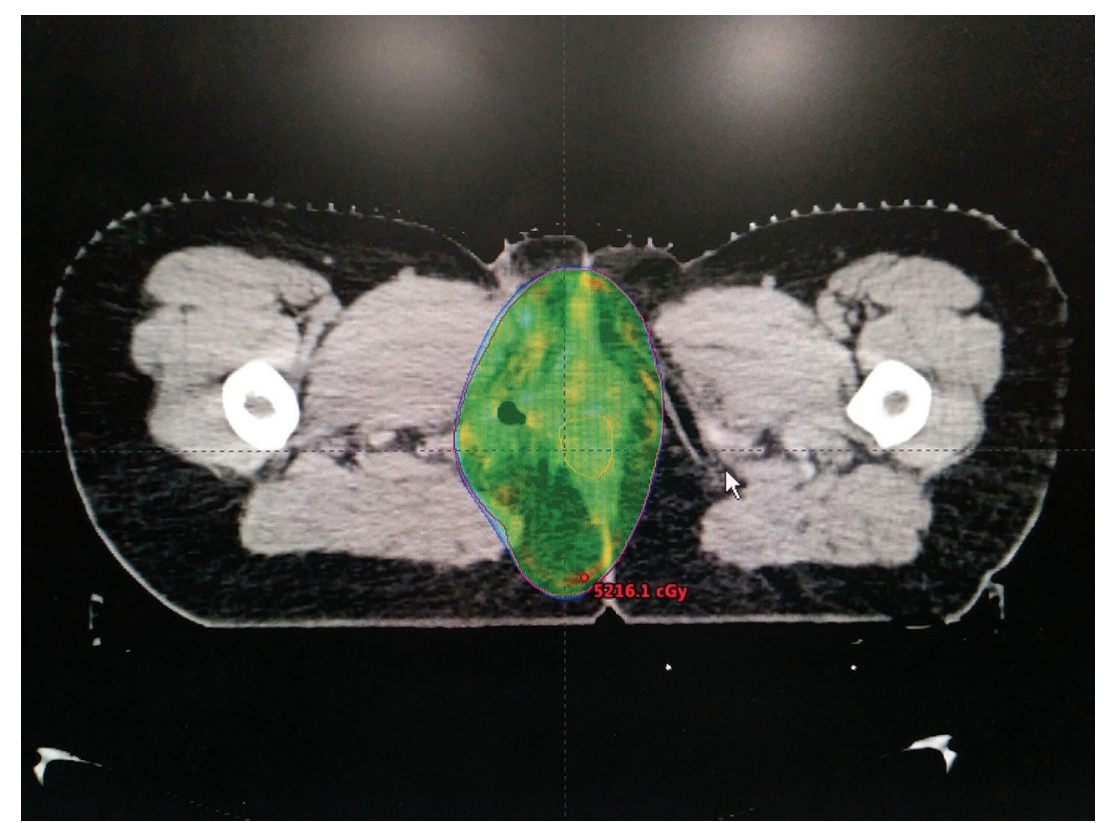

Fig. 2 Axial image of a VMATplan showing optimal coverage of the planning target volume by $95 \%$ of the prescribed dose. VMAT, volumetric modulated arc therapy.

described case) and magnetic resonance imaging (MRI). USG usually demonstrates a cystic or hypoechoic lesion..$^{15}$ On CT scan, the tumor may be hypodense to muscle, or may have both cystic and solid components. ${ }^{15}$ It has been suggested that MRI may be preferred due to characteristic swirling pattern observed and better depiction of tissue. ${ }^{15}$

Surgical management is the gold standard for both diagnosis and treatment of AA due to the aggressive and infiltrative nature of the disease. As it grows, AA tends to displace rather than invade adjacent organs, though invasion does occur in some cases. ${ }^{16}$ Complete surgical removal with negative margins is often not achieved in deeper pelvic tumors. ${ }^{17}$ Incomplete surgical removal is acceptable when significant morbidity is anticipated with broader excision. Recurrence rates range from 25 to $47 \%$, with $85 \%$ recurring within 5 years of initial surgery. ${ }^{7}$ Chemotherapy and RT have at present an undefined and limited role. Although AA is considered to be radioresistant, ${ }^{9}$ adjuvant RT has been reported to be effective in preventing recurrences. ${ }^{10}$ There is not a well-established role for RT in the treatment of AA, though there are a few cases described where radiation was utilized pre- or postoperatively to decrease tumor size or minimize risk of recurrence. ${ }^{7}$

AA has been shown to possess estrogen and progesterone receptors, suggesting growth is hormonally responsive. Use of hormone-modulating therapies has shown success in both neoadjuvant and adjuvant modality to prevent recurrence. Both postoperative radiation and GNRH-agonists have demonstrated complete resolution of residual or recurrent tumor growth and will continue to be valuable treatment options in residual and recurrent tumors. ${ }^{14,18}$

\section{Conclusion}

In conclusion, AA is a very rare disease with difficult diagnosis and lack of standard guidelines for treatment. Complete surgical excision of the tumor seems to be crucial. A multimodal approach is important. Even tumor-free margins do not prevent recurrence. Adjuvant RT has been reported to be effective in preventing local recurrences. Long-term follow-up is important because of repeated local recurrence.

\section{Funding}

None.

\section{Conflict of Interest}

None declared.

\section{References}

1 Smith HO, Worrell RV, Smith AY, Dorin MH, Rosenberg RD, Bartow SA. Aggressive angiomyxoma of the female pelvis and perineum: review of the literature. Gynecol Oncol 1991;42(1):79-85

2 Elchalal U, Lifschitz-Mercer B, Dgani R, Zalel Y. Aggressive angiomyxoma of the vulva. Gynecol Oncol 1992;47(2):260-262

3 Chen Lm, Schink JC, Panares BN, Barbuto D, Lagasse LD. Resection of a giant aggressive angiomyxoma in the Philippines. Gynecol Oncol 1998;70(3):435-439

4 Sun Y, Zhu L, Chang X, Chen J, Lang J. Clinicopathological features and treatment analysis of rare aggressive angiomyxoma of the female pelvis and perineum- a retrospective study. Pathol Oncol Res 2017;23(1):131-137

5 Ribaldone R, Piantanida P, Surico D, Boldorini R, Colombo N, Surico N. Aggressive angiomyxoma of the vulva. Gynecol Oncol 2004;95(3):724-728

6 Salman MC, Kuzey GM, Dogan NU, Yuce K. Aggressive angiomyxoma of vulva recurring 8 years after initial diagnosis. Arch Gynecol Obstet 2009;280(3):485-487

7 Sutton BJ, Laudadio J. Aggressive angiomyxoma. Arch Pathol Lab Med 2012;136(2):217-221

8 Nakamura T, Miura K, Maruo Y, et al. Aggressive angiomyxoma of the perineum originating from the rectal wall. J Gastroenterol 2002;37(4):303-308

9 Suit H, Spiro I. Radiation treatment of benign mesenchymal disease. Semin Radiat Oncol 1999;9(2):171-178 
10 Nyam DC, Pemberton JH. Large aggressive angiomyxoma of the perineum and pelvis: an alternative approach. Report of a case. Dis Colon Rectum 1998;41(4):514-516

11 Haldar K, Martinek IE, Kehoe S. Aggressive angiomyxoma: a case series and literature review. Eur J Surg Oncol 2010;36(4):335-339

12 Pereira P, Melo Abreu E, Cunha TM, Rolim I. Transperineal aggressive angiomyxoma. BMJ Case Rep 2017;2017:bcr-2016-217705

13 Wang YF, Qian HL, Jin HM. Local recurrent vaginal aggressive angiomyxoma misdiagnosed as cellular angiomyofibroblastoma: A case report. Exp Ther Med 2016;11(5):1893-1895

14 Bai HM, Yang JX, Huang HF, et al. Individualized managing strategies of aggressive angiomyxoma of female genital tract and pelvis. Eur J Surg Oncol 2013;39(10):1101-1108
15 Outwater EK, Marchetto BE, Wagner BJ, Siegelman ES. Aggressive angiomyxoma: findings on CT and MR imaging. AJR Am J Roentgenol 1999;172(2):435-438

16 Li X, Ye Z. Aggressive angiomyxoma of the pelvis and perineum: a case report and review of the literature. Abdom Imaging 2011;36(6):739-741

17 Chen $H$, Zhao H, Xie Y, Jin M. Clinicopathological features and differential diagnosis of aggressive angiomyxoma of the female pelvis: 5 case reports and literature review. Medicine (Baltimore) 2017;96(20):e6820

18 Shinohara N, Nonomura K, Ishikawa S, Seki H, Koyanagi T. Medical management of recurrent aggressive angiomyxoma with gonadotropin-releasing hormone agonist. Int J Urol 2004;11(6):432-435 